\title{
Quantum dynamics and Random Matrix theory
}

\author{
Hervé KUNZ* \\ Institute of theoretical Physics, Swiss Federal Institute of Technology Lausanne \\ Lausanne, CH-1015 EPFL, Switzerland
}

November 20, 2018

\begin{abstract}
We compute the survival probability of an initial state, with an energy in a certain window, by means of random matrix theory. We determine its probability distribution and show that is is universal, i.e. caracterised only by the symmetry class of the hamiltonian and independent of the initial state.
\end{abstract}

In classical mechanics, temporal chaos is caracterised by the extreme sensibility of a trajectory to variation of initial conditions. No direct analog of this phenomenon has been found in quantum mechanics so far. On the other hand, numerical evidence has been accumulated [1], showing that energy levels of a quantum system, whose classical counterpart is chaotic, have a statistical behavior described by Wigner's random matrix theory (RMT), on the mean level spacing scale. The question we want to adress is the following: are there specific predictions of RMT for quantum dynamics, which would caracterise the temporal behavior of "chaotic" quantum systems.

We consider the following situation: The system is prepared in an initial state $\varphi$ at time 0 , with an energy in a certain window, centered at $e$ and of width $2 s l(e)$, where $l(e)$ is the mean level spacing, and we want to compute the probability to find our system again in the state $\varphi$, at a later time $t$. This quantity that we call the survival probability $R$ is given by

$$
R=\left|\frac{\left(\varphi \mid \exp i \frac{t}{\hbar} H P(\Delta) \varphi\right)}{(\varphi, P(\Delta) \varphi)}\right|^{2}
$$

$H$ is the hamiltonian of our system and $P(\Delta)$ is the spectral projector on $\Delta$.

*hkunz@dpmail.epfl.ch 
We have chosen to take an energy in a range of the order of the mean level spacing in order to look at properties of the system which are independent of specific details. If $\left(\lambda_{j}, \psi_{j}\right)$ denote respectively the $j^{t} h$ eigenvalue and eigenvector of the Hamiltonian $H$, then the survival probability can be written as

$$
R=\left|\frac{\sum_{j=1} y_{j} \chi_{j} \exp 2 \pi i \tau x_{j}}{\sum_{j=1} y_{j} \chi_{j}}\right|^{2} \Theta\left(\sum_{j} \chi_{j}-1\right)
$$

where

$$
y_{j}=\left|\left(\varphi, \psi_{j}\right)\right|^{2}
$$

and if we define $x_{j}$ by the relation

$$
\begin{gathered}
\lambda_{j}=e+x_{j} l(e) \\
\chi_{j} \equiv \chi_{(-s, s)}\left(x_{j}\right)= \begin{cases}1 & \text { if }\left|x_{j}\right| \leq s \\
0 & \text { otherwise }\end{cases}
\end{gathered}
$$

The Heaviside function $\Theta$ ensures that there is at least one eigenvalue in $\Delta$. What appears naturally in this expression is the time measured in units of the Heisenberg time

$$
t_{H}=\frac{h}{l(e)}
$$

so that

$$
\tau=\frac{t}{t_{H}}
$$

If we look at this problem from the point of view of RMT, we will replace the Hamiltonian by a large $N \times N$ self-adjoint matrix, whose probability distribution is basis independent and therefore of the form

$$
e^{-W\left(\lambda_{1}, \ldots, \lambda_{N}\right)} d H
$$

Wigner's gaussian model corresponds to the choice

$$
W=\frac{N}{2} \sum_{j=1}^{N} \lambda_{j}^{2}
$$

The first conclusion to be drawn is that the survival probability is statistically independent of the initial state $\varphi$. This follows from the fact that the variables $\left\{y_{j}\right\}_{j=1}^{N}$ have a probability distribution, independent of $\varphi$ and given by:

$$
\mu_{N}(\mathbf{y}) d \mathbf{y}=\frac{1}{C_{N}} \delta\left(\sum_{j=1}^{N} y_{j}-1\right) \prod_{j=1}^{N} y_{j}^{\frac{\beta}{2}-1} d \mathbf{y}
$$

The parameter $\beta=1,2,4$ caracterise the symmetry class of the Hamiltonian, respectively orthogonal, unitary and symplectic. Equation (10) follows 
easily from the Haar measure on the corresponding groups. $C_{N}$ is a normalising constant.

The variables $\left\{x_{j}\right\}_{j=1}^{N}$ are statistically independent of the variables $\left\{y_{j}\right\}_{j=1}^{N}$ and have a distribution given by

$$
\frac{1}{D_{N}} \exp -W(e+\mathbf{x} l(e)) \Delta^{\beta}(\mathbf{x}) d \mathbf{x}
$$

where the Van der Monde determinant

$$
\Delta(\mathbf{x})=\prod_{1 \leq i<j \leq N}\left|x_{i}-x_{j}\right|
$$

comes from the change of variables $H_{i j} \rightarrow\left(\lambda_{j}, \psi_{j}\right)_{j=1}^{N}[2] . D_{N}$ is a constant of normalisation.

We can take $l(e)=\frac{1}{N \rho(e)}$, where $\rho(e)$ is the density of states when $N=\infty$. The problem that we need to solve now is to find the probability distribution of the survival probability $p(R) d R$ in the $N=\infty$ limit. We find that $R$ is not selfaveraging i.e. $p(R)$ is not a delta distribution concentrated on the mean value of $R$. On the other hand its probability distribution $p(R)$ is universal, i.e. it depends only on the symmetry parameter $\beta$, at least for a large class of $W$.

There are two formulas for $p(R)$, one more appropriate to small windows, another one to large windows.

In the first case, we decompose $p(R)$ into

$$
p(R \mid \tau)=\sum_{n=1}^{\infty} \frac{E_{n}}{1-E_{0}} p_{n}(R \mid \tau)
$$

where $E_{n}$ is the probability to find exactly $n$ eigenvalues in $\Delta$ and $p_{n}(R \mid \tau)$ is the conditional probability density of $R$ knowing that there are exactly $n$ eigenvalues in $\Delta$.

It can be expressed as

$$
\begin{gathered}
p_{n}(R \mid \tau)=\int_{-s}^{s} \hat{E}\left(x_{1}, \ldots, x_{n}\right) d^{n} x \int_{0}^{\infty} \mu_{n}\left(z_{1}, \ldots, z_{n}\right) d^{n} z \delta\left(R-\left|\sum_{j=1}^{n} z_{j} \exp 2 \pi i \tau x_{j}\right|^{2}\right) \\
\hat{E}\left(x_{1}, \ldots, x_{n}\right)=\frac{E\left(x_{1}, \ldots, x_{n}\right)}{E_{n}} \\
E_{n}=\int_{-s}^{s} E\left(x_{1}, \ldots, x_{n}\right) d^{n} x
\end{gathered}
$$

$E\left(x_{1}, \ldots, x_{n}\right)$ being the probability density of finding the $n$ eigenvalues in $\Delta$ at $\left(x_{1}, \ldots, x_{n}\right)$. 
Useful expressions for $E\left(x_{1}, \ldots, x_{n}\right)$ and $E_{n}$ can be found in [2] and [3]. It is expressible in terms of a determinant

$$
E\left(x_{1}, \ldots, x_{n}\right)=\operatorname{det} L_{\beta}\left(x_{i} \mid x_{j}\right) \quad ; \quad(i, j) \in(1 \ldots n)
$$

where

$$
L_{\beta}=\frac{K_{\beta}}{1-K_{\beta}}
$$

$K_{\beta}$ is an operator whose kernel in the simplest case $\beta=2$ is given by

$$
K_{\beta}(x \mid y)=\frac{\sin \pi(x-y)}{\pi(x-y)}
$$

defined on $L^{2}(-s, s)$.

Universality comes from the fact that $E\left(x_{1}, \ldots, x_{n}\right)$ is expressible in terms of the correlation functions and the latter ones depends only on $\beta$, for a large class of $W$. $W$ modifies only the density of states and therefore the mean level spacing $l(e)$.

This expression for $p(R \mid \tau)$ is mostly useful in the small window limit, because when $s \rightarrow 0$

$$
E_{n} \sim s^{\frac{\beta}{2} n^{2}+n\left(1-\frac{\beta}{2}\right)}
$$

Moreover in this case we have

$$
\lim _{s \rightarrow 0} s^{n} \hat{E}\left(s x_{1}, \ldots, s x_{n}\right)=A_{n} \prod_{1 \leq i<j \leq n}\left|x_{i}-x_{j}\right|^{\beta}
$$

so that the probability distribution of $R$ shows a scaling behavior

$$
\lim _{\substack{s \rightarrow 0 \\ R \rightarrow 1}} s^{-\beta-1} \operatorname{pr}\left\{\frac{1-R}{(\pi \tau s)^{2}} \geq x\right\}=\int_{x}^{1} g_{\beta}(\lambda) d \lambda
$$

the function $g_{\beta}(\lambda)$ being given by

$$
g_{\beta}(\lambda)=A_{\beta} \lambda^{\frac{\beta-1}{2}}\left[\frac{1}{2} \sqrt{1-\lambda}+\frac{1}{2} \ln \lambda-\ln 1+\sqrt{1-\lambda}\right]
$$

On the other hand, one can see from eq (13) and (14) that the probability distribution of $R$ is well defined at infinite times. Namely

$$
p_{n}(R \mid \tau)=p_{n}(R \mid \infty)+O\left(\frac{1}{\tau}\right)
$$

as can be seen by an integration by parts where

$$
p_{n}(R \mid \infty)=\int_{0}^{\infty} \mu_{n}\left(z_{1}, \ldots, z_{n}\right) \int_{0}^{2 \pi} \prod_{j=1}^{n} \frac{d \phi_{j}}{2 \pi} \delta\left(R-\left|\sum_{j=1}^{n} z_{j} e^{i \phi_{j}}\right|^{2}\right)
$$


Using an integral representation for the delta appearing in the definition (10) of the $\mu_{n}$, we can reexpress (25) as

$$
p_{n}(R \mid \infty)=\frac{1}{4 \pi c_{n}} \int_{\epsilon-i \infty}^{\epsilon+i \infty} d u e^{u} \int_{0}^{+\infty} d r r J_{0}(\sqrt{R} r)\left[\int_{0}^{\infty} d z e^{-u z} J_{0}(r z) z^{\frac{\beta}{2}-1}\right]^{n}
$$

$\epsilon$ being any positive number, and $J_{0}(x)$ the Bessel function. This expression can be simplified, considerably when $\beta=2,4$.

In the unitary case $(\beta=2)$ one finds

$$
p_{n}(R \mid \infty)=\frac{n-1}{2}(1-R)^{\frac{n-3}{2}}
$$

For a large window of energy, it is more appreciate to find another expression for $p_{n}(R \mid \tau)$. It is given as some integral over a Fredholm determinant $\mathcal{G}$.

$\mathcal{G}$ is a generating function for the variables $\left\{y_{j}\right\}$ and $\left\{x_{j}\right\}$ appearing in the definition of $R$, eq (2).

$$
\left.\mathcal{G}(\nabla ; \varphi ; \ddagger)=\lim _{\mathcal{N} \rightarrow \infty}\langle\exp -\rangle \mathcal{N} \sum_{\mid=\infty}^{\mathcal{N}} \dagger_{\mid} \chi_{\mid}\left[\nabla \cos \left(\in \pi \tau \S_{\mid}+\varphi\right)+\ddagger\right]\right\rangle
$$

It can be expressed in terms of the operator $K_{\beta}$ appearing in eq (19), when $\beta=1,2$ as

$$
\left.\mathcal{G}=\mathcal{E}_{l}\left[\operatorname{det}\left(\infty+\mathcal{K}_{\beta}\right\}^{\frac{\beta}{\epsilon}}\right)\right]^{\frac{\beta}{\epsilon}}
$$

with

$$
E_{0}=\left[\operatorname{det}\left(1-K_{\beta}\right)\right]^{\frac{\beta}{2}}
$$

and $g$ is the multiplication operator by the function

$$
g=\left[1+\frac{2 i}{\beta}[z+r \cos (2 \pi \tau x+\varphi)]\right]^{-1}
$$

When the window is large $(s>>1)$ we can expand the determinant in powers of $K_{\beta}$, the first two terms of this expansion dominating the other ones [4].

One finds that the probability distribution is exponential.

$$
\lim _{s \rightarrow \infty} \frac{1}{s} p\left(\frac{R}{s} \mid \tau\right)=\frac{1}{\sigma(\tau)} \exp -\frac{R}{\sigma(\tau)}
$$

In the orthogonal case $(\beta=1)$, for example

$$
\sigma(\tau)= \begin{cases}4-2|\tau|+|\tau| \ln 1+2|\tau| & \text { if }|\tau| \leq 1 \\ 2+|\tau| \ln \frac{2|\tau|+1}{2|\tau|-1} & \text { if }|\tau| \geq 1\end{cases}
$$

One can notice the singularity at the Heisenberg time $\tau=1$ and the fact that $\sigma(\infty)$ exists. 
However if we smooth out in time $R(\tau)$, taking for example

$$
\bar{R}=\frac{1}{\tau_{1}-\tau_{0}} \int_{\tau_{0}}^{\tau_{1}} R(\tau) d \tau
$$

then we get a selft-averaging quantity

$$
\lim _{s \rightarrow \infty} \frac{1}{s} p\left(\frac{\bar{R}}{s}\right)=\delta(\bar{R}-\bar{\sigma})
$$

with

$$
\sigma=\frac{1}{\tau_{1}-\tau_{0}} \int_{\tau_{0}}^{\tau_{1}} d \tau \sigma(\tau)
$$

Some numerical work on chaotic billiards [5], in the large window limit, confirm this exponential distribution. Integrable billiards show a very different behaviour [5].

Finally, we would like to mention the fact that Wigner's energy level statistics can be obtained for models, where eigenvalues and eigenvectors are correlated. We think therefore that the study of quantum dynamics could discriminate between such models and those we have considered where they are uncorrelated.

\section{Acknowledgements}

This work is dedicated to F.Y. Wu for his $70^{\text {th }}$ birthday.

\section{References}

[1] See for example the review by O. Bohigas, Les Houches 1989, Chaos et physique quantique (M-J. Giannoni et al, North Holland, 1991).

[2] M.L. Mehta, Random Matrices (Ed. Academic Press Inc, 1991).

[3] C.A. Tracy and H. Widom, Journal of Statistical Physics 92, 809 (1998)

[4] H. Kunz, J. Phys. A : Math \& Gen 32, 2171 (1999)

[5] R. Aurich and F. Steiner, Int. J. Mod. Phys. 13, 2361 (1999) 